\title{
6 The success view situated
}

I have developed the success view against the background of two views: the simple conditional analysis on the one hand and possibilism on the other. There was good reason for this way of setting the stage. Those two views really are the background for the contemporary debate on abilities. Virtually every contemporary account of abilities draws on resources that are provided by one or both of these two background views, and the struggle to overcome the shortcomings of the background views shines through every single one of them.

Yet, there are quite a few more recent views on the market, and those views, of course, need to be taken into account as well. This chapter turns to this task. My aim, in what follows, is to situate the success view among some of the recent views on abilities, point out similarities and differences, and provide reasons for favoring the success view over the other accounts.

Most of the recent interest in abilities stems from two debates that are taking place in different areas of philosophy. On the one hand, there is the so-called "new dispositionalism" (Vihvelin 2004, 2013; Smith 2003; Fara 2008). As noted before $(\rightarrow 5.4)$, new dispositionalists think that abilities are dispositions of sorts. Of course, that idea is not very new (Ryle 1949; Moore 1912). What makes new dispositionalism new is the combination of that idea with two further claims.

First, new dispositionalists share the conviction that understanding the link between abilities and dispositions provides an immediate and new route to compatibilism about freedom and determinism. In chapter 5.4, I argued that the line of reasoning leading up to this conviction is inconclusive.

The second conviction characteristic of the new dispositionalism is more important for the points that will concern us in the course of this chapter: new dispositionalists unanimously reject the simple conditional analysis of dispositions. According to the simple conditional analysis of dispositions (Moore 1912; Ryle 1949), an object has a disposition to $M$ when $C$ (to dissolve when placed in liquid, say) if and only if the object would $\mathrm{M}$, if $\mathrm{C}$ were the case (if it would dissolve, if it were placed in liquid).

Just as in the case of abilities, it is nowadays widely believed that dispositions cannot be analyzed in terms of a single counterfactual. In part, the reasons for the rejection of this view are analogous to problems that have also played a role in our discussion of the simple conditional analysis of abilities. Just like abilities, dispositions can be masked, come in degrees, and ascriptions of dispositions are correspondingly context sensitive. My ability to fall asleep at night can be masked by too much street noise (Fara 2005: 50); one thing can be

2 OpenAccess. (c) 2020 Jaster, published by De Gruyter. (cc) BY-NC-ND This work is licensed under the Creative Commons Attribution-NonCommercial-NoDerivatives 4.0 License.

https://doi.org/10.1515/9783110650464-007 
more fragile than another (Manley \& Wasserman 2008: 72); and what counts as fragile in a discussion among engineers need not count as breakable in the context of a discussion among ceramists (ibid.).

What we can note, then, is that a single counterfactual is just as ill-suited when it comes to accounting for dispositions as it is when it comes to accounting for abilities. New dispositionalists are well aware of this fact. That is why they reject the simple conditional analysis.

Which view of dispositions do they endorse instead? Here, new dispositionalists diverge. The new dispositionalists are not primarily interested in a positive account of dispositions. Their primary concern is to provide an account of abilities in terms of dispositions. Only sometimes is that account then supplemented by a positive account of dispositions.

Thus, new dispositionalist views about abilities come in different granularities, if you so will. On the coarse-grained level, new dispositionalists provide an analysis of abilities in terms of dispositions. Thus, Fara suggests that "[a]n agent has the ability to $\phi$ in $\mathrm{C}$ if and only if she has the disposition to $\phi$ when, in $\mathrm{C}$, she tries to $\phi$ " (Fara 2008: 848). ${ }^{1}$ And Vihvelin writes that "[t]o have an ability is to have a disposition or a bundle of dispositions" (Vihvelin 2004: 431). ${ }^{2}$ On the coarse-grained level, then, new dispositionalists are more or less d'accord on how to analyze abilities. ${ }^{3}$

On a more fine-grained level, this need not be the case. New dispositionalists need not, and do not, agree on how to analyze dispositions. Thus, new dispositionalist views on abilities can, and do, differ once various different views on dispositions are plugged into the coarse-grained analysis. On a more finegrained level, then, new dispositionalist views on abilities can, and do, take rather different forms, depending on the account of dispositions which is endorsed over and above the coarse-grained analysis of abilities in terms of dispositions.

How does the success view relate to the coarse-grained and various existing and potential fine-grained versions of the new dispositionalism? On the face of it, my views are fully in line with the coarse-grained new dispositionalist view

1 Fara's view may look a lot like the conditional analysis, but is to be read differently. See fn. 62 . 2 That Vihvelin speaks about bundles is rooted in the fact that she distinguishes between basic abilities - roughly: abilities to perform basic actions - and complex abilities - roughly, abilities to perform complex actions. Basic abilities correspond directly to individual dispositions, on her view. Complex abilities correspond to bundles of dispositions (2004: 431).

3 Vihvelin (personal conversation) thinks that only what I have called agentive abilities can be analyzed in terms of the disposition to $\phi$ when trying to $\phi$. Non-agentive abilities correspond to other dispositions, on her view. 
that abilities are dispositions of sorts. As I laid out in chapter 5.6, I find it very plausible that

DISPOSITION. an object has a disposition to $\phi$ if and only if the object $\phi$ 's in a sufficient proportion of the relevant trigger situations for $\phi$-ing.

Combine this plausible analysis with my view that having an ability to $\phi$ is for an agent to $\phi$ in a sufficient proportion of a subset of the relevant possible S-trigger situations and what you get is the new dispositionalist's credo that abilities simply are special instances of dispositions.

Note, however, that I am not analyzing abilities in these terms. I am not committing myself to the view that abilities are dispositions. Instead, I am committing myself to a certain analysis of abilities - the success view - and to the further hypothetical statement that if dispositions are analyzable along the lines of DISPOSITION - which I take to be plausible, but by no means certain - then abilities turn out to be dispositions of sorts. Thus, I may be in line with the new dispositionalist credo that abilities are dispositions, but I am in no way committed to it.

The coarse-grained new dispositionalist view of abilities will not concern us any further in the present chapter. We will, however, be concerned with a few analyses of abilities that emerge when one plugs different analyses of dispositions into the coarse-grained new dispositionalist picture that abilities are dispositions of sorts. More specifically, we will look the sophisticated conditional analysis which is obtained by analyzing abilities in terms of Manley and Wasserman's (2008) very powerful account of dispositions $(\rightarrow 6.2)$ and the view one obtains by analyzing abilities in terms of Vetter's (2015) extended possibilist account of dispositions $(\rightarrow 6.3)$.

Let me now proceed to talk about the other strand of literature in which abilities have played an important role quite recently: the literature emerging from the so-called virtue epistemology movement.

"Virtue epistemology" is an umbrella term for a rather wide variety of views, which differ quite substantially in many ways. According to one strand of those views, abilities have to be considered to be at the heart of epistemic properties, such as knowledge or justification. Among the virtue epistemologists who phrase their views in terms of abilities, there have been serious attempts to understand that notion better. Let me give you a little bit of background on that.

As diverse as virtue epistemological accounts are on many levels, there are two basic commitments that unify all of them (Greco \& Turri 2015). The first is that virtue epistemologists consider epistemology to be a normative discipline. 
In Greco and Turri's words, that "implies that epistemologists should focus their efforts on understanding epistemic norms, value and evaluation” (2015).

Different virtue epistemological accounts spell this out differently. While all virtue epistemologists agree that epistemic norms, value, and evaluation play a crucial role in understanding epistemological concepts, there is a divide between virtue epistemologists who believe that those concepts cannot be analyzed in purely non-normative terms (e.g. Zagzebski 1996, 2009) and those who think that they can (e.g. Greco 1999, 2009; Sosa 2007).

The second commitment common to all virtue epistemological views is that "intellectual agents and communities are the primary source of epistemic value and the primary focus of epistemic evaluation" (Greco \& Turri 2015). Greco and Turri explain the general line of thought by way of a very helpful example:

An evidentialist might define an epistemically justified belief as one that is supported by the evidence, and then define evidence in a way that entirely abstracts from the properties of the person. On such an approach, it would be natural to understand intellectual virtues as dispositions to believe in accordance with the evidence (which, again, is defined independently, without mentioning the virtues). A virtue epistemologist would reverse the order of analysis, defining justified belief as one that manifests intellectual virtue, and evidence in terms of intellectual virtue. (2015)

With this very brief introduction into the two main ideas of virtue epistemology in the background, let us see how abilities enter the picture.

Abilities play a central role in one very prominent strand of virtue epistemology which stands in the tradition of reliabilist accounts of knowledge. On those virtue epistemological views, knowledge is non-accidentally true belief, where this, in turn, is spelled out in terms of belief that is true because it has been obtained in an intellectually virtuous way and not by mere coincidence (Sosa 1991; Greco 2009).

What is it for a belief to turn out right by reason of virtue and not just by coincidence? Here, many virtue epistemologists have spelled out their view by using the notion of an ability. Greco, for instance, lays out that

[t]o say that someone knows is to say that his believing the truth can be credited to him. It is to say that the person got things right due to his own abilities, efforts and actions, rather than due to dumb luck, or blind chance, or something else. (Greco 2003: 111)

That a belief is obtained via one's intellectual or cognitive abilities is what guarantees, according to the above-mentioned virtue epistemologists, that the belief is non-accidental. That is because an ability to $\phi$ is had only if the agent's success in $\phi$-ing is reliable enough. This is where the reliabilist intuition underlying 
these virtue epistemological views becomes visible. According to reliabilists (e.g. Goldman 1979), one knows that $\mathrm{p}$ only if one's belief that $\mathrm{p}$ is obtained through a reliable process. The virtue epistemological twist of this intuition is the postulate that one's beliefs be formed in virtue of one's intellectual abilities.

It should now be clear why it is quite important to supplement one's virtue epistemological view that knowledge is true belief obtained through ability with an actual account of what it is to have an ability. In order to show that abilities have to do with reliability, one obviously needs to talk about abilities quite a bit.

It is therefore unsurprising that two very promising accounts of abilities have come up in the virtue epistemological sphere. One is put forward by Greco (2007), the other one by Sosa (2015). Despite their shared virtue epistemological background, however, the views are not equivalent. Greco's view is neutral on a question that is addressed by Sosa. And while Greco's view is fully compatible with the success view, it takes a bit more effort to see how exactly the success view relates to Sosa's view of abilities: Sosa's view happens to be the very same view one obtains when plugging Manley and Wasserman's view of dispositions into the new dispositionalist credo that abilities just are dispositions of sorts. Despite the fact that the new dispositionalism and virtue epistemological accounts differ very substantially in their objectives, there are actually overlaps in the views of abilities that have emerged against these two backgrounds.

In what follows, I will not systematize the views of abilities that are going to be discussed along the new dispositionalism/virtue epistemology divide. And in fact, not all of the views I will discuss have emerged from one of those two background debates. Thus, I will focus on the views themselves and systematize them in accordance with their relation to the success view.

We will begin, in the next section, 6.1, with Greco's analysis of abilities in terms of success, which is neutral regarding some crucial details and thus compatible with a variety of other views, including the success view itself. In fact, I fully agree with Greco's view of abilities and see the success view as the proper means to spell out the account more fully.

In section 6.2, we'll move on to what I will call "the sophisticated conditional analysis". The sophisticated conditional analysis is modeled on Manley and Wasserman's (2008) account of dispositions. Moreover, it is defended by Sosa (2015), who is a virtue epistemologist and, in a more complex version, by Vihvelin (2013), who is a new dispositionalist. Enriched with some ameliorations, I will argue, the view has the same merits as the success view. But the reason for that is that the sophisticated conditional analysis cum ameliorations simply is a version of the success view. Since it is a less elegant and somewhat misleading one, I suggest we should nevertheless endorse the success view instead. Yet, the views 
are really very similar, and I view its proponents as allies in most crucial respects.

In section 6.3, we'll look at a view that has not actually been defended by anyone but is obtained by analyzing abilities along the lines of Vetter's (2015) account of dispositions. Since the resulting view is quite interesting from a new dispositionalist perspective, but also, and especially, in relation to the success view, I take it to be a fruitful endeavor to take a look at it in some detail and see why it will not do the job.

In section 6.4, finally, we'll look at a view which has recently been put forward by Maier. Maier's view has certainly been developed with an eye towards free will issues, but in contrast to new dispositionalist views of abilities, it does not emphasize any ideas about the relationship between abilities and dispositions. Instead, Maier thinks that abilities should be analyzed in terms of the choice theoretic notion of an option. Doing so strikes me as unfortunate in a number of ways. Accounting for abilities in terms of options, it seems to me, really gets some things wrong that can be accommodated for very smoothly by the success view.

Despite the fact that I substantially disagree with some of the views I'll discuss and think we should favor the success view over all of them, let me say one thing right away. In principle, I consider all of the views I will be discussing in this chapter as attempts to voice most of the core insights that I have been trying to voice in the form of the success view. The recent views on abilities do not differ vastly from the success view, or, for that matter, from one another. All of them, for instance, incorporate the idea that having an ability is a matter of performing (or, in the case of Maier's view, having options) across a certain proportion of worlds or situations. Regarding very many fundamental aspects, there is considerable agreement between proponents of the various views I will talk about. The devil is in the details. Let's see if we can pin it down.

\subsection{Success 1.0}

Let's start with Greco's view on abilities as developed in connection with his virtue epistemological endeavor to analyze knowledge in terms of agents' intellectual abilities. On Greco’s view,

SUCCESS_1.0. $\mathrm{S}$ has an ability $\mathrm{A}(\mathrm{R} / \mathrm{C})$ relative to environment $\mathrm{E}=$ Across the set of relevantly close worlds $\mathrm{W}$ where $\mathrm{S}$ is in $\mathrm{C}$ and in $\mathrm{E}, \mathrm{S}$ has a high rate of success in achieving R. (Greco 2007: 61) 
I choose the label SUCCESS_1.0, because Greco's view is not fully compatible with the success view; rather, it voices the same basic intuition, albeit without filling in the details. The success view, as I have presented it, is a 2.0-version of Greco's account, so to speak.

To see that, let's look at SUCCESS_1.0 in some more detail. First, note that Greco is concerned with abilities relative to environments. Thus,

Jeter has an ability to hit baseballs relative to typical baseball environments. He has no such ability relative to active war zones ... where he lacks the concentration needed to hit baseballs (ibid.).

Moreover, Greco points out, "abilities are tied to a set of relevant conditions" (ibid.). Thus, "it does not count against Jeter's having the ability [to hit baseballs] ... that he can't hit baseballs in the dark, or with sand in his eyes" (ibid.: 60).

I have to say that it is not quite clear to me how environments and conditions are supposed to differ. Greco emphasizes that there is considerable overlap between the two. The tentative difference he has in mind is just that environments are supposed to be "relatively stable circumstances", whereas conditions are "sets of shifting circumstances within an environment" (ibid.). Let's grant him that the distinction can be made sense of. Nothing hinges on this in what follows.

It should be obvious that Greco's emphasis on conditions and environments sits well with the success view. The success view postulates that an agent has an ability if and only if she $\phi$ 's in a sufficient proportion of the relevant possible situations in which some S-trigger for $\phi$-ing is present. The crucial notion here, of course, is that of the relevant possible situations. The relevant possible situations can be selected as to include only those in which the agent is in a typical baseball environment in which the agent does not have sand in her eyes, for instance. Of course, these situations will have to be otherwise similar enough to the actual world in many respects. We always hold certain features of the agent and the world fixed across the relevant possible situations. Greco distributes these two onto two distinct elements of his analysis. He talks about abilities relative to environments and analyzes those abilities in terms of relevantly close worlds.

I take it that which way one goes here is by and large a matter of taste. Let me emphasize, however, that it is often important to drop the restriction to a certain environment and conditions when thinking about abilities. That is because while it may not count against an agent's ability simpliciter in some context that the agent is unable to exercise it in extremely challenging circumstances, it may 
well count against a certain degree of her ability. If Jeter hits baseballs even with sand in his eyes or in active war zones (or both!), while his buddy Jeff doesn't, then this counts in favor of Jeter being better at hitting baseballs than Jeff. By restricting one's focus on abilities relative to environments and conditions right from the outset, one is in danger of losing sight of the fact that for some purposes it is useful to drop the restrictions to environments in order to compare success rates across environments. This is a minor point, though. By and large, SUCCESS_1.0 is on the right track.

Secondly, note that Greco uses "rate of success" in the same way I have used it throughout the book: ${ }^{4}$ as a modal notion. What matters is success across possible worlds:

[To say that someone has a high rate of success]is to say both more and less than that they have a good track record with respect to achieving that result. ... Actual track records can be the result of good luck rather than ability. Likewise, actual track records can be the result of bad luck rather than lack of ability. (ibid.: $60 \mathrm{f}$.)

Note that Greco speaks of possible worlds instead of possible situations. I take this to be merely sloppy and not a matter of dispute, however. Once we start looking at the rate of success, we need to look at the rate of success even within worlds. Alternatively, we will have to use "world" to mean "centered world" or the like. I don't think Greco would dispute that. Again, SUCCESS_1.0 is in line with the success view of ability.

We can note, then, that everything Greco says (or can be assumed to mean by what he says) is fully compatible with the success view as I have been advancing it in this book. But - and this is why I have been referring to it as the 1.0 version of the success view itself - it is less informative in one crucial respect. Greco postulates, roughly, that to have an ability, the agent has to be successful across sufficient range of situations (or worlds, as Greco puts it). What Greco is silent about, however, is what exactly this amounts to.

The answer to this question is by no means obvious. Greco's view is, for instance, compatible with a view according to which having an ability is a matter of $\phi$-ing in a sufficient proportion of the relevant worlds, full-stop. No restriction to intention cases here. What we are evaluating, on this view, is just the proportion of the $\phi$-ing situations among the totality of the situations in which the relevant facts obtain. Call this view "possibilism+". It is obtained by analyzing abilities along the lines of Vetter's (2015) account of dispositions. Vetter does not defend this view, because she sees that it results in problems, and I will walk

4 In fact, I have borrowed the term from him. 
you to these problems in section 6.3. What is important for our concerns here is just that possibilism+ is compatible with Greco's SUCCESS_1.0.

Greco's view is also compatible with a view, according to which having an ability is a matter of a multitude of counterfactuals being true, each of which has the form "If some S-trigger for $\phi$-ing were present and $\mathrm{C}$ were the case then S would $\phi$ ”, where C picks out some more or less fully specified situation. Call this view "the sophisticated conditional analysis". Versions of it are defended by Sosa (2015) and Vihvelin (2013), and an analogue of the view has been defended by Manley and Wasserman (2008) in the case of dispositions.

There are many ways to put flesh to the bones of Greco's account, then, and the success view offers one of those ways. Just like possibilism+ and sophisticated conditional analyses, then, the success view can be seen as a more elaborate account that is fully in line with Greco's basic view, but by no means entailed by it.

\subsection{The sophisticated conditional analysis}

Having identified Greco's view as fully compatible with the success view, let's move on to a view which emerges when analyzing abilities along the lines of a very powerful account of dispositions that has recently been proposed by Manley and Wasserman (2008). On Manley and Wasserman's view, which I will refer to as "the sophisticated conditional analysis of dispositions",

SOPH_D. $\mathrm{N}$ is disposed to $\mathrm{M}$ when $\mathrm{C}$ if and only if $\mathrm{N}$ would $\mathrm{M}$ in some suitable proportion of C-cases. (ibid.:76)

SOPH_D is quite interesting for our concerns, because it owes its potency to the fact that it solves all of the most pressing problems of the simple conditional analysis of dispositions. The view accounts smoothly for masks, degrees, and context sensitivity. Since these are all issues that will have to be accounted for in the case of abilities as well, there is good reason to think that an analogue of SOPH_D will also prove powerful in the realm of abilities.

In what follows, I'll argue that this is indeed so. But only after adding a few crucial supplements to the sophisticated conditional analysis, and only because the sophisticated-conditional-analysis-cum-supplements will turn out to be nothing other than a less elegant and somewhat misleading version of the success view itself. It should therefore not come as a surprise that the two views are eventually on a par when it comes to their merits.

To get a better grip on SOPH_D, let's look at the view in some more detail. First, let's try and understand what C-cases are. C-cases, according to Manley 
and Wasserman, are first of all cases in which a proper stimulus is present. For solubility, C-cases are those in which $\mathrm{N}$ is placed in a liquid; for fragility, C-cases are cases in which $\mathrm{N}$ is subjected to force; for irascibility, C-cases are cases in which $\mathrm{N}$ is provoked. Some dispositions have manifold stimuli; in that case, the C-cases comprise cases in which any of those stimuli is present. Other dispositions do not seem to have a specific stimulus to begin with - in that case the Ccases comprise cases of any kind. Talkativity, for instance, is simply the disposition to talk, not the disposition to talk in certain situations. A subject is talkative, therefore, on Manley and Wasserman's account if and only if the subject talks in a suitable proportion of all cases (ibid.: 72).

All cases? Not absolutely all. For apart from the stimulus being present, there are two more important features of C-cases. First, C-cases will have to have the same laws of nature. That seems right. The fact that a thing breaks upon being hit or dissolves upon being placed in liquid in worlds in which the laws are different from ours does not count towards the thing's fragility and solubility, respectively. The actual laws will have to be fixed across the Ccases.

Secondly, C-cases will have to be such that the object retains its intrinsic properties (ibid.: 76). With their focus on intrinsic properties, Manley and Wasserman are in good company - dispositions are usually thought of as a matter of the intrinsic properties of their bearers. ${ }^{5}$ Thus, fragility seems to be a matter of a certain molecular structure, and irascibility seems to be a matter of a certain brain structure. And these need to be fixed across the C-cases.

So much for the C-cases. Let's now look at SOPH_D itself in some more detail. Like the simple conditional analysis, SOPH_D analyzes dispositions by means of counterfactuals. However, SOPH_D differs from the simple conditional analysis in one very important way. The simple conditional analysis analyzes dispositions in terms of a single counterfactual. If the stimulus occurred, the manifestation would follow. SOPH_D, in contrast, analyzes dispositions in terms of a very large number of counterfactuals with highly specific antecedents, each of which specifies a something close to a complete situation. ${ }^{6}$ Thus, to be irascible, say, a person will have to meet a sufficient proportion of counterfactuals of the

5 Recently, a plausible case has been made for the existence of extrinsic dispositions (McKitrick 2003). If that is right, then the $C$-cases will sometimes comprise extrinsic features as well.

6 In a critical discussion of the view, Vetter (2011) suggests that there is, in fact, a different interpretation available, but I'll neglect that second interpretation for the time being, since Vetter's suggestion pretty much is to interpret PROP_D in terms of the success view, which is also what I will suggest later on. 
form "If $\mathrm{C}$ were the case, then the object would freak out", where $\mathrm{C}$ will specify a different situation in each counterfactual.

This difference between the simple conditional analysis and SOPH_D is crucial. In contrast to the simple conditional analysis, what matters for SOPH_D is not just what is the case in the stimulus worlds that are overall closest to the actual world. Instead, pretty much all possible stimulus cases in which the laws and the object are as in actuality matter. If the counterfactual "If $\mathrm{C}$, then $\mathrm{N}$ would M" is true for a suitable proportion of the C-cases, so understood, then the object (or agent) has the disposition. If not, it (or she) lacks it.

How does this solve the problems that beset the simple conditional analysis? Well, the problem of degrees and context sensitivity is solved because, as Manley and Wasserman point out,

[h]ow big a proportion is 'suitable' will depend not only on the dispositional predicate involved but also on the context of utterance. (...) We can think of the contexts as providing the standards for 'fragility' by establishing a requisite proportion of C-cases in which an object would break, for example. In particular, for something to count as 'fragile' in the mouth of the chemist it must break under a much larger proportion of the sorts of stress conditions relevant to fragility. (ibid.)

This should sound familiar. Manley and Wasserman account for degrees in the very same way as the success view: by means of proportional quantification over cases, where the context determines which proportion of cases is required in a context for the ascription of the disposition to apply simpliciter $(\rightarrow 4.3)$. Unlike in the case of the success view, the cases go into the antecedents of counterfactuals, of course, but the basic mechanism for degrees and context sensitivity is the same in both cases.

The solution for making cases is likewise familiar. As Manley and Wasserman lay out,

the right-hand side of our bi-conditional holds even if an object happens to be in 'bad' case where its disposition is (....) masked. All that is required is that the object would break in a suitable proportion of stimulus cases, where these will include any (...) masking cases; and it makes no difference whether these are actual. (ibid.: 76f.)

Here, too, the strategy is basically the same as in the case of the success view ( $\rightarrow$ 4.6). Since the cases over which the quantification ranges are so diverse that the mask will not be present in most of them, intentions and performances will match up in a suitable proportion, even if a mask is present in the actual world.

When I introduced SOPH_D, I said that the C-cases will be highly specific; they will specify something close to a complete situation. We are now in a posi- 
tion to see why. The presence or absence of any potential mask of a disposition has to be settled in the antecedent of each counterfactual. That is why the antecedents of the counterfactuals Manley and Wasserman postulate in their account have to be so specific that they specify, for every potential mask, whether it is present or not. The antecedents of the counterfactuals we obtain that way are highly specific indeed. They specify the situation more or less fully.

Having said all this, let's now return to our original topic: abilities. Obviously, Manley and Wasserman's contribution to the dispositions debate is highly relevant for our concerns in that connection. Let's consider a Manley and Wasserman inspired account of abilities and see how it fares. Here is the view. Call it "the sophisticated conditional analysis of abilities":

SOPH_A. An agent S has an ability to $\phi$ if and only if S would $\phi$ in a suitable proportion of C-cases.

What are the C-cases this time? Well, first of all, they will be cases in which an Strigger is present. ${ }^{7}$ That, after all, is the analogue to the stimulus in the case of mere dispositions, and it is crucial to Manley and Wasserman's view of dispositions that the C-cases are stimulus cases. Thus, SOPH_A will have to be read as the view that an agent has an ability if and only if there is a suitable proportion of highly specific C-cases for which the counterfactual "If $\mathrm{C}$ were the case, then the agent would $\phi$ " comes out true, where each C-case is such that an S-trigger is present. $^{8}$

Note that I am already building an important insight into this interpretation of the C-cases which goes far beyond Manley and Wasserman's template of the view. As we will see later on in this section, proponents of the sophisticated conditional analysis, such as Sosa, think of the C-cases as cases in which the agent is properly motivated to $\phi$. But as we know by now, this is unfortunate, because it fails to account for non-agentive abilities.

What more can we say about the C-cases? Again, we have to move beyond Manley and Wasserman's template here. Manley and Wasserman's insight that the C-cases will be cases in which the laws of nature are held fixed surely applies in the case of abilities as well. But that is not the only restriction that will have to be imposed on the C-cases. As we know from our discussion of general and spe-

7 As in the case of the simple conditional analysis, one can in principle tinker with the motivational state in the antecedent. For reasons of unity, I will again focus on intentions.

$8 \mathrm{I}$ am again presupposing that Manley and Wasserman intend their view to be understood in terms of a multitude of counterfactuals and postpone Vetter's (2011) remark that their view may just as well be understood along the lines of the success view. 
cific abilities, abilities are had in view of the relevant fact, where it will vary which facts are relevant. Sometimes, we will want to know what an agent can do in view of her total situation; sometimes, in view of her location; sometimes, in view of who is helping; sometimes in view of her intrinsic make-up; and so on.

To accommodate this insight within the framework of the sophisticated conditional analysis, whatever facts go into the "in view of" phrase will have to be fixed across the C-cases. As we know, this means that what is held fixed will vary across contexts and cannot be specified in terms of a single set of rules.

Thus, the C-cases will have to be thought of as cases in which an S-trigger is present, the laws are as in actuality, and the relevant properties of the agent whatever they may be in a particular context - are held constant. To make this entirely clear, let us refine SOPH_A in the following way:

SOPH_A'. An agent $S$ has an ability to $\phi$ if and only if $S$ would $\phi$ in a suitable proportion of the relevant S-trigger cases (the $\mathrm{C}$-cases), where it will vary across contexts which S-trigger cases are relevant.

This, then, is our sophisticated conditional analysis of abilities. It derives from the new dispositionalist assumption that abilities are dispositions, combined with Manley and Wasserman's view about dispositions and supplemented with some of the crucial insights about abilities that we have gained in the course of this book.

Sophisticated conditional analyses have recently been proposed, in different versions, by Sosa (2015) and Vihvelin (2013). Sosa, as I read him, advances something very close to SOPH_A'. Sosa writes:

What then is required for possession of a competence? Required for archery competence, for example, is a sufficient spread of possible shots (covering enough of the relevant shapes and situations one might be in as an agent) where one would succeed if one tried, an extensive enough range. (Sosa 2015: 98)

As I read this admittedly sketchy passage, what Sosa lays out here is that for an agent to have an ability, the counterfactual "If the agent tried, she would succeed" has to turn out true for a multitude of possible shots which cover various situations in which the agent may be. This is very close to what Manley and Wasserman lay out for dispositions and to what I have suggested as an analogue of their view for abilities.

Note that Sosa is concerned with an agentive ability in this passage - the ability to hit a target. Thus, he does not phrase his view in terms of S-triggers. For the example he discusses, that is fine. When it comes to abilities quite gen- 
erally, the view will have to be stated in terms of S-triggers. Once it is thus reformulated, it just is what I spelled out under the heading SOPH_A' above.

Note, too, that Sosa is quite aware of the fact that the realm of the C-cases will vary across contexts. He postulates that the possible shots will have to cover "enough of the relevant shapes and situations one might be in as an agent” (Sosa 2015: 98, my emphasis). And which shapes and situations are relevant? That will vary. To see that, note that Sosa distinguishes between three types of competence:

(a) the innermost driving competence: that is, the structural seat in one's brain, nervous system, and body, which the driver retains even when asleep or drunk [= seat], (b) the fuller inner competence, which requires also that one be in proper shape, i.e., awake, sober, alert, etc., [= shape] and (c) the complete competence or ability to drive well and safely, which requires also that one be situated with control of a vehicle, along with appropriate road conditions pertaining to the surface, the lighting, etc. [= situation] (ibid: 96f.)

It does not matter for our purposes here whether or not the three types of competence Sosa outlines are exhaustive. What is important is that Sosa is very explicit on his view that while a condition like being tied down, say, "does not affect the inner [seat + shape] competence, nor the innermost [seat] competence", it "does bear on one's complete [seat + shape + situation] competence" (ibid.: 98).

This is interesting, because, plausibly, the way the view about competence Sosa advances delivers all of those uses of "competence" is by restricting the relevant shapes and situations one might be in as an agent he talks about when he formulates his view on competences as a whole. Sosa is thus well aware of the fact that the C-cases will have to be varied in accordance with the context of an ability ascription.

Vihvelin advances a somewhat different view. Brushing over some of the details, however, it becomes clear that, structurally, she defends something very close to SOPH_A' as well. Vihvelin argues that

VIHV. S has the narrow ability at time $\mathrm{t}$ to do $\mathrm{R}$ as the result of trying if and only if, for some intrinsic property B that $\mathrm{S}$ has at $\mathrm{t}$, and for some time $\mathrm{t}$ ' after $\mathrm{t}$, if $\mathrm{S}$ had the opportunity at $\mathrm{t}$ to do $\mathrm{R}$ and $\mathrm{S}$ tried to do $\mathrm{R}$ while retaining property $\mathrm{B}$ until time t', then in a suitable proportion of these cases, S's trying to do R and S's having of B would be an S-complete cause of S's doing R (Vihvelin 2013: 187).

This is quite an unwieldy condition, but for current purposes, we can focus on the structural features of the view. As I read Vihvelin (and I may be mistaken here), Vihvelin's condition is just an enriched version of SOPH_A' with a narrower focus. The focus is narrower because Vihvelin is exclusively interested in what 
she calls "narrow abilities" - abilities that are held in virtue of an agent's intrinsic properties. The view is an enriched version of SOPH_A', because those abilities are then analyzed in terms of a multitude of rather complex counterfactuals roughly of the form

* If S were in a (relevant?) case in which S had the opportunity and tried and had intrinsic property B, then the trying and B would cause S's doing R.

As in the case of SOPH_A', a sufficient proportion of those counterfactuals will have to come out true. Structurally, then, VIHV is just another version SOPH_A'.

An obvious difference, of course, is that Vihvelin (as I read her) postulates further requirements for a case to qualify as a C-case. Apart from being such that the agent tries to $\phi$ in it (or, as I suggest, some S-trigger for $\phi$-ing is present), a case will also have to provide the agent with an opportunity and some intrinsic property of the agent will have to be retained in order for it to qualify as a C-case. But these latter two requirements are just additional layers of complexity and can be neglected for what is to come. Everything I say in what follows applies to VIHV just as much as it applies to the basic version of the view as put forward by Sosa.

So much for the exposition of the sophisticated conditional analysis of abilities. Let's now move on to scrutinizing it. The view is promising - that much is clear. As in the case of dispositions, masks, degrees, and context sensitivity are unproblematic. I don't think we have to go through that in detail. SOPH_A handles these things analogously to the way SOPH_D does, which means that it uses the same basic tools that also play a crucial role in the success view. I trust that the reader has a good understanding of these issues at this stage in the book.

The real problem lies elsewhere. Just like the simple conditional analysis, SOPH_A' runs into the problem of impeded intentions. To yield the right verdict, it should be possible for SOPH_A' to be not met in such cases, thus yielding the very prevalent sense in which a coma patient, say, cannot raise her arm. But does SOPH_A' yield this result? Not as far as I can see.

According to SOPH_A', an agent has such an ability if and only if a multitude of counterfactuals of the form "If $\mathrm{C}$ obtained, $\mathrm{S}$ would $\phi$ " are true. "C" specifies one particular C-case in each counterfactual, and each of those Ccases will be an S-trigger case. In the case of an agentive ability like the ability to raise one's arm, it will be an intention case. Thus, what SOPH_A' postulates is that an agent has the ability to raise her arm if and only if a multitude of counterfactuals of the form "If $S$ intended to raise her arm and the case is otherwise relevant (and thus a C-case), S would raise her arm” are true. 
But now a problem looms. Either the coma is held fixed across the C-cases or it is not. As I will argue in what follows, SOPH_A' is - wrongly - satisfied in both cases. This may not be obvious at first, because at first sight, holding the coma fixed seems like a very promising move. The problem with the simple conditional analysis, after all, was that it always considers the closest intention worlds and that those worlds are located beyond the worlds in which the agent is in a coma to begin with. The antecedent catapulted us too far out, as it were.

The same does not seem to happen if the coma is held fixed across the Ccases. Since the agent is in a coma in all C-cases, the C-cases will comprise only situations in which the agent is as impeded as in actuality. This promises to yield - rightly - that SOPH_A' will not be satisfied in the coma case. If the antecedent of each counterfactual comprises the fact that the agent is in a coma, then one expects that no consequent will comprise the agent's arm raising. There will therefore not be any true counterfactuals of the form "If $\mathrm{C} \mathrm{ob-}$ tained then the agent would raise her arm”. Consequently, it seems that SOPH_A' will not be met.

But as good as it sounds, this line of thought is mistaken. In the coma case, the C-cases comprise only coma cases, that much is true. But apart from that, the C-cases have to meet some other criteria, too. Most importantly, they will have to be cases in which the agent intends to $\phi$ (in the case of agentive abilities). And here we encounter a problem. For as we know, there simply are no such cases to begin with. No coma case is an intention case.

In the framework of the success view, this insight plays a key role in the treatment of cases of impeded intentions. The success view (of agentive abilities) states that the agent has to $\phi$ in a sufficient proportion of relevant intention cases. In cases of impeded intentions, there are no relevant intention cases, because no coma case is an intention case. And that is good. For if there are no relevant intention cases, then the proportion of arm raising cases among the relevant intention cases is unspecified and a forteriori not sufficiently high. The condition postulated in the success view therefore rightly fails to be met in such a case.

The same is not true in the case of SOPH_A', however. For at least on the standard semantics of counterfactuals, a counterfactual comes out vacuously true if there are no antecedent worlds (Lewis 1973: 16). Thus, the proportion of C-cases for which it is true that the agent would raise her arm, if that case obtained is actually very high. For all C-cases, the counterfactual will be satisfied. Thus, SOPH_A' yields the verdict that the coma patient has the ability to raise 
her arm. The obvious sense in which she cannot raise it fails to be accounted for. ${ }^{9}$

Vihvelin's more complex version of SOPH:A' is not better off. On Vihvelin's view, recall, the narrow, i.e. intrinsic, ability to raise one's arm is analyzed in terms of a multitude of counterfactuals of the form "If S were in a (relevant?) case in which $S$ has the opportunity to raise her arm and tried to raise her arm and had intrinsic property B, then the trying and B would cause S's raising her arm, where $C$ is a placeholder for various circumstances.” Again, the antecedent seems to be impossible. Holding the coma fixed (either in the form of intrinsic property B or in the form of looking only at coma-circumstances), there seem to be no opportunity worlds left.

Or are there? Let's allow for that; an opportunity for $\phi$-ing may simply be a situation in which nothing external conflicts with $\phi$-ing, in which case a coma patient may well have an opportunity to raise her arm. Even then, though, the antecedent will come out impossible. Holding the coma fixed, there will not be any worlds left in which the agent tries to $\phi$. Despite all complexity, the basic problem remains.

Can this consequence be avoided, if we vary the coma? Unfortunately not. First of all, it is entirely unclear why varying the coma should be permitted in the first place. The sophisticated conditional analysis can only deliver an account of general and specific abilities if the context fixes the set of facts that have to obtain throughout the C-cases. Whatever determines the relevant worlds in the framework of the success view goes into the C-cases in the framework of the sophisticated conditional analysis. That's the mechanism one needs, if the distinction between general and specific abilities is to be made sense of. It is hard to see how one could invariably vary the coma without losing the capacity to give a convincing account of general and specific abilities.

Even if the coma (and other impediments of the same kind) were varied, however, this would not solve the problem of impeded intentions. Varying the coma channels us back to the problem of the impeded intention as it arose for the simple conditional analysis: when the coma is varied across the C-cases, then there is no reason to assume that the agent would not raise her arm in a suitable proportion of $\mathrm{C}$-cases. The coma would be treated like a mask on that account. While present in the actual world, it is varied across the C-cases. In masking cases, SOPH_A' yields - rightly - that the agent has the masked ability. In cases of impeded intentions, this is the wrong outcome. Thus, no matter how we twist and turn the view, impeded intentions remain troublesome for the con-

9 Sosa seems to be oblivious to this problem (cf. Sosa 2015: 98). 
ditionalist - the increased sophistication of SOPH_A' does not seem to be of much help here.

Yet, that is not to say that SOPH_A' is doomed. For of course, there is one simple move open to the proponent of the view that has also proved advisable for proponents of the simple conditional analysis. The proponent of SOPH_A' can simply supplement her view by adding a further condition that is explicitly designed to avoid the unwelcome consequences in the coma case and other cases of impeded intentions.

To see how, let's return to the first horn of the dilemma once more. The problem here was that, since the C-cases have to be both coma cases and cases in which the agent intends to $\phi$, there will simply be no C-cases to begin with. All counterfactuals of the form "If $S$ intended to $\phi$ and the case is otherwise relevant (and hence a coma case), S would $\phi$ ”, will come out vacuously true, because the antecedent specifies an impossible state of affairs. Hence, SOPH_A' fails to account for the very prominent sense in which the coma patient lacks the ability to raise her arm or get up from her bed.

The proponent of SOPH_A' can avoid this problem by adding a supplementary postulate to SOPH_A': the C-cases have to be possible states of affairs in the first place. In cases in which the coma is held fixed across the C-cases, this supplementary postulate is obviously not met. Hence, the so supplemented view yields, rightly, that the coma patient lacks the ability to raise her arm in such cases. (Of course, the coma does not have to be held fixed in every context. It may well be varied, in which case the supplemented view yields, again rightly, that the coma patient has the ability to raise her arm. This is all as before. The problematic sense for the original SOPH_A' is just the one in which it is true that the coma patient cannot raise her arm.)

The supplemented view I suggest should replace SOPH_A' is therefore the following:

SOPH_+. An agent $\mathrm{S}$ has an ability to $\phi$ if and only if (i) $\mathrm{S}$ would $\phi$ in a suitable proportion of the relevant S-trigger cases (the C-cases), where it will vary across contexts which S-trigger cases are relevant, and (ii) there are possible S-trigger cases (Ccases) to begin with.

Thus understood, cases of impeded intentions, such as the coma case, are rendered unproblematic. Even though condition (i) of SOPH_+ is met, condition (ii) is not when the coma is fixed across the C-cases.

But then isn't SOPH_+ just as powerful as the success view? After all, it seems to have the very same merits. Is it just a matter of taste which view one adopts? As far as I can see, the answer is a qualified yes. The success view 
and $\mathrm{SOPH}+$ + both account for the adequacy conditions and they don't run into the problems that proved destructive for the simple conditional analysis or possibilism.

The reason for that, however, is that $\mathrm{SOPH}_{-}+$is the success view in counterfactual guise. First of all, as should have become clear in the previous discussion, the program of the sophisticated conditional analysis only gets off the ground if some key elements of the success view are integrated. And secondly, as will become clear in a moment, once those elements are integrated, the two views actually turn out to be almost equivalent.

To see that, recall that the success view states that for an agent to have an ability to $\phi$, the agent has to $\phi$ in a sufficient proportion of the relevant possible situations in which the agent intends to $\phi . \mathrm{SOPH}_{-}+$states that for an agent to have an ability to $\phi$, "If $\mathrm{C}$ obtained, $\mathrm{S}$ would $\phi$ " has to be true for a sufficient proportion of C-cases, where those C-cases in turn are relevant possible situations in which $S$ intends to $\phi$.

The only difference between the two views is that the success view postulates that a sufficient proportion of C-cases are $\phi$-ing cases, whereas $\mathrm{SOPH}_{-}+$ postulates that a sufficient proportion of $\mathrm{C}$-cases be such that the counterfactual "If $\mathrm{C}$ were the case, $\mathrm{S}$ would $\phi$ " holds for them. At first sight, that seems to make a difference, logically speaking. But insofar as each C-case is a fully specified situation, the two views are in fact equivalent. And as we saw above, the Ccases will in fact have to be (almost) fully specified situations in order for masks to be accounted for.

If that is so, then $\mathrm{SOPH}_{-}+$and the success view turn out virtually equivalent. To say that a sufficient proportion of the relevant C-cases are such that the counterfactual "If $\mathrm{C}$ were the case, $\mathrm{S}$ would $\phi$ " turns out true is only to say that a sufficient proportion of the relevant $\mathrm{C}$-cases are $\phi$-ing cases. The counterfactual only turns out true for some C-case, after all, if that C-case is a $\phi$-ing case. That's just what happens when you insert whole situations into the antecedent of a counterfactual. You do violence to the counterfactual by turning it into a material conditional. ${ }^{10}$

Speaking of violence, let me now tell you why I think the success view, as I have stated it in this book, is to be favored over the counterfactual rendering of the same idea in terms of SOPH_+. I don't see why one should use counterfactuals in the statement of the view, if the counterfactuals don't actually function as counterfactuals. Nostalgia could be one reason: "The simple conditional analysis

10 As far as the C-cases are not fully, but almost fully specified situations, there is a logical difference between the views, but that difference is minuscule. 
looked so promising, so let's see how we can tweak the framework until we eventually get a version that works." Personally, I don't find this motive very convincing, but if you are strongly attracted to counterfactuals (even the somewhat perverted ones figuring in $\mathrm{SOPH}_{-}+$), be my guest and cling to them. If you are rather more of a non-sentimental type, I take it you are better off with the success view as I have suggested it. When it comes right down to it, the counterfactuals in SOPH_+ are a scam. Let's be straightforward. Let's endorse the success view instead.

\subsection{Possibilism+}

In this section, let us look at a view we get by analyzing abilities along the lines of Vetter's (2015) account of dispositions. This is a fruitful thing to do, because the limitations of the resulting view of abilities will once more shed light on the importance of the success rate as spelled out by the success view.

Vetter's project in her book is to reduce modality in general - including metaphysical modality - to the potentialities of objects and agents. Paradigmatic instances of potentialities, on her view, are dispositions. Even though Vetter does not endorse possible worlds metaphysically, she views possible worlds semantics as useful enough to state an analysis of what it is for an agent to have a disposition along the following lines:

POSS+_D. An object $\mathrm{x}$ has a disposition to $\phi$ if and only if $\mathrm{x} \phi$ 's in a sufficient proportion of the relevant possible situations. ${ }^{11}$

Let's call this view "possibilism+ about dispositions". Being soluble, on this view, is for something to dissolve in a sufficient proportion of the relevant worlds; being irascible is for someone to freak out in a sufficient proportion of the relevant worlds.

With this view, Vetter is in obvious opposition to all kinds of views about dispositions which stand in the tradition of the conditional analysis, because Vetter does not believe, and she is very explicit about that (Vetter 2011), that dispositions are individuated, even just in part, by their stimuli. On her view, they are individuated exclusively by their manifestations.

In this respect, she is fully in line with the ideas of Kratzer $(1977,1981)$ and others, who treat disposition ascriptions in the same way as they treat ability as-

11 Vetter speaks of worlds where I used the term "situations", but what she means by that is just what I mean by "situations". 
criptions: as possibility statements. On that view, which we can call "possibilism about dispositions", for a thing to be soluble is for it to be possible for that thing to dissolve. This, in turn, is spelled out in terms of possible worlds: for $p$ to be possible is for there to be a relevant world in which $\mathrm{p}$ is the case. So far, nothing new; possibilism about dispositions is analogous to possibilism about abilities as we have discussed it at length in chapter 3.

Of course, Vetter does not endorse possibilism. Possibilism about dispositions faces some of the same problems that possibilism about abilities faces too. Like abilities, dispositions come in degrees and they are context sensitive, for instance (Manley \& Wasserman 2008). Possibilism about dispositions is just as unapt to account for those features as possibilism about abilities is.

For this reason, Vetter squares the possibilist framework with the insight that having a disposition is a matter of proportions. In doing so, she incorporates one crucial element that is also part of the success view and of the sophisticated conditional analysis. The degree of an object's disposition to $\phi$ corresponds, on Vetter's view, to the proportion of the relevant possible situations, in which the object $\phi$ 's among the relevant possible situations as a whole. This allows her to account for degrees and context sensitivity.

While degrees and context sensitivity are accounted for by the same means as in the case of the sophisticated conditional analysis and the success view, however, there is one very crucial difference between Vetter's and these other two views. Vetter sticks closer to possibilism in that she clings to her conviction that dispositions are individuated by their manifestation alone.

The sophisticated conditional analysis $(\rightarrow 6.2)$ and my own suggestion of how to analyze dispositions $(\rightarrow 5.4)$ both put crucial emphasis, not just on the manifestation of dispositions, but on the stimulus as well. The sophisticated conditional analysis looks at a multitude of counterfactuals of the form "If $\mathrm{x}$ had been in C, $\mathrm{x}$ would have $\phi$-ed", where C-cases are, among other things, cases in which a stimulus is present. The view I suggested, DISPOSITION, looks at the relevant possible situations in which a stimulus is present and evaluates whether or not the proportion of the $\phi$-ing situations among those is high enough.

The same is not true for POSS+_D. POSS+_D restricts the possible situations to the relevant ones - the ones in which the relevant facts obtain - and varies whether or not the stimulus occurs. The relevant facts are facts about the object; usually, its intrinsic properties. Everything else is varied. And while DISPOSITION postulates that the proportion of the $\phi$-ing cases has to be high enough among the relevant stimulus cases, POSS+_D postulates that the proportion of the $\phi$-ing cases has to be high enough among the relevant possible situations as a whole. The proportion that matters is therefore a different one. 
In what follows, I will not discuss Vetter's account of dispositions. Vetter's book is very rich, and I could not possibly do it justice in the course of this section. I will therefore content myself with a few remarks about the relationship between Vetter's account of dispositions and my own views on abilities and dispositions at the end of this section. Primarily, however, I want to focus on whether Vetter's account of dispositions also provides a viable framework for abilities. As I will argue, it does not.

Applying Vetter's thinking about dispositions to abilities, what we get is the following view:

POSS+_A: An agent S has an ability to $\phi$ if and only if S $\phi$ 's in a sufficient proportion of the relevant possible situations.

Let's call this view "possibilism+ about abilities”. Let me point out very clearly right away that Vetter does not endorse this view. In fact, she rejects it, and partly for reasons I am going to discuss in a moment. Yet, POSS+_A is an interesting suggestion, and one worth exploring, because it is so closely related to the success view and will once more shed light on the importance of the success rate the modal tie between intentions (or other S-triggers) and performances postulated by the success view.

As is easy to see, POSS+_A follows the same scheme as POSS+_D. Having an ability to $\phi$, just like having a disposition to $\phi$, is for the agent to $\phi$ in a sufficient proportion of the relevant possible situations. No mention of intentions (or any other S-trigger). Analogously to the case of POSS+_D, it will vary, across the relevant possible situations, whether or not an intention is formed. What one checks for is the proportion of the $\phi$-ing cases to the relevant possible situations as a whole.

The relevant possible situations, of course, are the situations in which the relevant facts are held fixed, and as we know from our discussion of general and specific abilities, it will have to vary which facts are relevant in a given context. Sometimes, we hold only stable, mostly intrinsic facts about the agent fixed; sometimes, we hold lots of facts about the external situation fixed as well. We do not, however - and this is very important when it comes to the difference between POSS+_A and the success view - restrict the relevant possible situations to the ones in which an intention is formed.

What we have here, then, is a view that resembles the success view very closely. Subtract the restriction to intention situations from the success view and what you end up with is POSS+_A. That makes POSS+_A a very interesting view to look at in some detail. If POSS+_A comes out right, then the success view would turn out unnecessarily complex. Why bother with intentions and the suc- 
cess rate, if focusing on the ratio of performances among the relevant possible situations as a whole does the job just as well?

As we will see in what follows, however, POSS+_A does not come out right. There is good reason for focusing on the success rate and not, as POSS+_A suggests, on the ratio of performances to the totality of the relevant possible situations. For simplicity, let us focus on agentive abilities in what follows.

First of all, I take it to be highly unintuitive to look at the proportion of $\phi$-ing cases in relation to all relevant possible situations, given that the agent will not intend, not have reasons, and not desire in any way to $\phi$ in the vast majority of those relevant possible situations. Think of my general ability to hit a particular bull's eye; one in a bar in Sweden, say. I have that ability. I have it in view of a variety of my features. Let's focus on the ability in view of one set of features: my brain structure and muscular constitution. Now, according to POSS+_A, I have the ability to hit that bull's eye in Sweden, because I hit it in a sufficient proportion of situations in which my brain structure and my muscular constitution is as in actuality.

But just think of how incredibly small that proportion is going to be! In the vast - let me say it again: vast! - majority of all situations in which those features are as in actuality, I don't have the slightest intention or reason to go about hitting the bull's eye in a bar in Sweden. In fact, I will not be in Sweden in most situations and will not have the slightest reason or intention to hit the bull's eye in that bar. POSS+_A, however, factors all of those situations in when it comes to determining whether or not I have the ability to hit that bull's eye. It looks at the proportion of hitting situations in relation to all situations in which my brain structure and muscular constitution is as in actuality.

Now think about differences in the degree of an ability. According to POSS +_A, someone with a high degree of the ability under consideration and someone with a low degree of that ability differ with respect to the proportion of hitting cases among the situations in which their brains and muscles are as in actuality. But given how minuscule the proportions are in each case, the difference will be almost unspeakably tiny.

The same is true for POSS+_D, by the way. Take solubility. Sugar has that disposition in view of its molecular structure. According to POSS+_D, this is because there is a sufficient proportion of dissolution cases among the relevant possible situations - the situations in which sugar has its actual molecular structure. Note, however, that in the vast majority of those situations, the sugar is not placed in any kind of liquid. Thus, the proportion of dissolving cases among the relevant possible situations will be minuscule, and so will differences between highly soluble, moderately soluble, and almost insoluble substances. 
This is not a knock-down argument against POSS+_D or POSS+_A. But it raises doubts as to whether the proportion the views postulate are really what matters. In what follows, I will focus exclusively on POSS+_A and argue that the proportion it postulates is certainly not the right one. Consequently, POSS +_A fails. As we'll see, the reasons for that failure speak very much in favor of the success view.

That the proportion POSS+_A postulates is the wrong one can be seen by looking at a case Vetter herself puts forward against POSS+_A (ms.). Take an obsessive-compulsive agent, whose intrinsic psychological constitution is such that the agent forms the intention to wash her hands every five minutes. Compare that agent to a psychologically sane person, who only washes her hands in standard situations. We can assume that both agents are equally good at washing their hands - they have the hand washing ability to the same degree. Their psychological constitution does not make a difference in this regard.

The problem is that POSS+_A predicts otherwise. That is because, holding each agent's psychological constitution fixed, the proportion of hand washing situations is going to be much higher in the case of the obsessive-compulsive agent than in the case of the sane agent. And hence, the obsessive-compulsive agent should come out as having the ability to a higher degree. (The example may not be ideal; degrees of the ability to hand wash are usually almost exclusively evaluated on the level of achievement. To see that this does not affect the argument, though, I invite you to run the same argument with an obsessive-compulsive dart player instead.)

What does the argument show? First, of all, I submit, it shows that there is something wrong with POSS+_A. Perhaps you are not willing to draw that conclusion right away. Perhaps you are inclined to say "Wait a minute. Why can't the proponent of POSS+_A simply resist your move of holding the psychological constitution of the agent fixed? The obsessive-compulsive agent does not have the ability to wash her hands to a higher degree than any average person, because each agent's ability to wash their hands has nothing to do with their respective psychological constitutions. Why should the proponent of POSS+_A allow for you to fix an arbitrary set of features to evaluate whether or not the agent has the ability to wash her hands?"

In response, let me first of all clarify one thing. I fully agree that it is not legitimate to fix an arbitrary set of features to evaluate whether an agent has some ability. It is not, for example, legitimate to fix my broken arm in evaluating whether or not I have the ability to do a handstand in view of my muscular constitution. Likewise, it is not legitimate to fix the fact that I am asleep in evaluating whether or not I have the ability to sing in view of my larynx system. What is legitimate, however, is to fix my broken arm in evaluating my ability to do a 
handstand in view of my broken arm, and to fix the fact that I am asleep in evaluating my ability to sing in view of my being asleep.

Casting a look back at the argument against POSS+_A, you will note that the ability under consideration there is the ability to wash one's hands in view of one's psychological constitution. To evaluate whether or not and to what degree an agent has that ability, we are not only allowed to, but we actually have to hold the agent's psychological constitution fixed. And doing so, we get wrong results. Thus, there is something wrong with POSS+_A.

But perhaps it is illegitimate to look at the ability to wash one's hands in view of one's psychological constitution from the outset? What kind of ability is that supposed to be after all? Shouldn't the proponent of POSS+_A resist focusing on weird abilities like that right from the outset? Differently put, shouldn't she resist the idea that any set of facts go into the "in view of" phrase?

In response to this way of framing the objection, I would like to stress two points. First of all, I really do think, and I wish to commit myself to this with respect to the success view as well, that any set of facts can go into the "in view of" phrase of any ascription of the form "S can, in view of F, $\phi$ ”. Our view should yield the right results, no matter whether we look at the ability to sing in view of one's larynx system or the ability to sing in view of the Chinese economy crisis or in view of the amount of hair on my head or in view of the fact that your favorite color is red and I have never been in Korea. There is nothing wrong with those kinds of abilities. They are weird, all right. But there are no good grounds for rejecting them. Facts that don't have anything to do with an ability should be safely insertable into the "in view of" phrase and not make any difference to the agent's having or lacking them. This is not the case when it comes to POSS+_A.

The second, and perhaps even more important point is that the ability to wash one's hands in view of one's psychological constitution is not a "weird" ability to begin with. It is in view of her psychological constitution, after all, that someone phobic of water does not have the ability to wash her hands. She does have that ability in view of many other features: in view of her strength and motor control she has it, for instance. But there is a very clear sense in which she lacks the ability to wash her hands. To account for that sense, we need to be looking at her ability to wash her hands in view of her psychological constitution. The ability to wash one's hands in view of one's psychological constitution is a perfectly legitimate ability. I thus take it that the argument above really does show that something is wrong with POSS+_A.

But what exactly is wrong with it? I submit the trouble stems from the fact that the proportion of hand washing situations to the totality of relevant possible situations is not the proportion that matters. What really matters is the proportion of hand washing situations to the relevant possible situations in which 
the agent intends to wash her hands. And that is just what the success view states. Thus, the case speaks very much in favor of imposing an additional restriction to intention situations upon the relevant ones as a whole.

The success view can explain very smoothly why the obsessive-compulsive agent is not better at washing her hands than any average agent: her success rate is not higher. The ratio of situations in which the obsessive-compulsive agent washes her hand among the relevant possible situations in which she intends to do so is just about the same than the same ratio in the case of the sane person. This bolsters my claim that it is the modal success rate which matters when it comes to (degrees of) abilities. Without the further restriction to intention situations, counterexamples arise.

At least in the case of abilities, then, there is strong reason to think that an individuation solely in terms of the manifestation - or, rather, the exercise - is on the wrong track. Abilities, it seems, have to be analyzed by a ratio which ties intentions (or S-triggers quite generally) to exercises. The success view postulates just that.

In doing so, the success view does not run into the unintuitive consequence of minuscule proportions either, by the way. The proportions the success view postulates are usually quite high. Typically, an agent who is good at something will more often than not succeed in doing that thing upon intending to do it. Within the framework of the success view, minuscule proportions are the exception, not the rule, if someone has an ability.

\subsection{An option-based account}

Maier (2013) is concerned with a certain class of properties, which he refers to as the agentive modalities. The agentive modalities, as Maier understands them, include options, abilities, skills, and potentialities. They are modal properties of some agent, which involve some relation to the agent, and in virtue of which certain "can" statements are made true. Agentive modalities are the truth makers of statements of the form "S can A" where " $\mathrm{S}$ " denotes the agent and " $\mathrm{A}$ " refers to some act-type (Maier 2013: 2).

As Maier lays out, there are two different kinds of questions one may ask about agentive modalities. On the one hand, there are what he calls external questions - questions about the relationship between agentive modalities and other modal properties, such as the question whether or not agentive modalities are reducible to other modal properties or whether the metaphysics of modality will "require a species of modality that is distinctively agentive" (ibid.). On the other hand, there are what he calls internal questions, which concern the rela- 
tionship between agentive modalities themselves. "Are they miscellany, or a unified collection? If the latter, what is it that unifies them?” (ibid.).

With respect to external questions, Maier remains officially neutral (ibid.:10), although he formulates strong reserves concerning a reductive enterprise (ibid.:7). What he is explicitly concerned with are the internal questions. Here, Maier offers an account, according to which all agentive modalities can be elucidated in terms of the most basic among them. Maier calls them options. He writes:

There are at least two ways in which a given agent may be able to perform a certain action. It may be now in her power to perform that action: there is, as it were, nothing between her and the deed. This corresponds to what I have been calling options. Alternately, she may be able to perform the action, though it is not now in her power to do so. For example, Rachel may be able to speak Mandarin even though, under the effects of novocaine, it is not now in her power to do so. Let us call this latter way of being able to perform an action a general ability. (Maier 2013: 11)

The set-up of the example should ring a bell: options seem to be specific abilities. ${ }^{12}$ But they seem to be specific abilities of a special kind. As Maier has it, options are "the species of agentive modality that figures in the framing of choice situations" (ibid.:8). This makes options particularly well-suited as a fundament for an account of agentive abilities, in Maier's view, because they have two important features: they are "[d]eterminate in the sense that it is perfectly clear, in some given case, whether or not this agentive modality obtains" (ibid.:7). And they are "[i]ndispensable in the sense that, in our theorizing about agency, this agentive ability is one that we cannot do without" (ibid.:7).

Why think that options have those two features? According to Maier, they have them in virtue of their role in decision theory. Options are indispensable, because questions of rationality or morality cannot be formulated without them. In order to be able to ask what some agent ought to do, we need to compare the (moral or rational) value of her options (ibid.:8f.). And they are determinate, because otherwise the recommendations of decision theory could not be determinate. Since recommendations like "Take the option that maximizes overall utility" are perfectly determinate, however, so has to be the notion of an option (ibid.: 9).

From here, it is only a small step to Maier's full account of general abilities. For general abilities can now be defined in terms of options:

12 Maier has confirmed this in personal conversation. 
MAIER. S has the general ability to $\mathrm{A}$ if and only if $\mathrm{S}$ has, in a suitable proportion of similar circumstances, the option of A-ing. (ibid.: 19)

This yields the following picture. On the one hand, there are options which play an indispensable role in moral theory and decision theory and are well-understood by their theoretical role in the framing of choice situations. Options are presumably basic in the sense that their modality cannot be reduced any further. On the other hand, there are general abilities which are defined in terms of options. To have a general ability is to have an option in a suitable proportion of similar circumstances. ${ }^{13}$

So much for the exposition of Maier's view. Let's now see how the view relates to the success view and what to make of it. Some similarities between Maier's account and the success view should be obvious. First, Maier explicitly endorses Kratzer's insight that abilities are always had in view of certain facts. That is why he restricts the possible circumstances to the similar ones. We obtain the similar circumstances by holding certain facts fixed. Which facts we hold fixed can vary. In this respect, Maier's view resembles the success view rather closely.

Yet, I take the success view to be one step ahead. The success view speaks of relevant possible situations where Maier speaks of similar circumstances. The reason is that we may sometimes be interested in what the agent can do given certain hypothetical circumstances. As I have laid out earlier $(\rightarrow 4.5 \mathrm{f})$ the relevant possible situations will in that case contain some that are dissimilar to the actual world in crucial respects. Maier's account fails to provide the resources for cases like that. The success view is more flexible in that respect.

The second obvious similarity between Maier's view and the success view is that both analyze abilities in terms of the notion of a suitable proportion of circumstances. The proportions that matter are different, of course. Maier counts the options among the similar circumstances, while the success view counts performances among the relevant intention cases, but the general idea of applying proportional quantification to cases is obviously the same. Moreover, Maier does not work with the idea of a weighted proportion, but obviously he can avail himself of that tool as well. Basically, the success view and Maier's view are on a par here.

The merits of the proportional quantification are the same in both cases, too. Proportions deliver an account of degrees, and the ability simpliciter is had if and only if the degree of the ability is high enough. Thus, an agent's degree of an ability is higher, on Maier's view, the higher the proportion of option cases

13 The class of the agentive modalities is wider. I will focus exclusively on abilities, though. 
among the similar circumstances is. Apart from the fact that Maier is interested in a different proportion, this looks a lot like the account of degrees I developed in 4.3 .

So much for the similarities between Maier's and the success view. Let's now turn to the crucial difference: the central role of options within Maier's framework. According to Maier, what matters for an agent's ability to $\phi$ is the proportion of the agent's options to $\phi$ among the similar circumstances. Options, in turn, are defined functionally. They are "the species of agentive modality that figures in the framing of choice situations” (ibid.:8). Here, Maier's view encounters some severe problems.

First, and most importantly, I don't believe the notion of an option is actually as well-understood as Maier seems to think. In telling us that options are "the species of agentive modality that figures in the framing of choice situations" (ibid.:8), Maier makes it seem as though this would settle the issue once and for all. In reality, though, choice theory knows of various ways to define options, some of which are obviously not what Maier has in mind.

Choice theory is very often understood as a psychological model, or as Wolfgang Schwarz puts it, "as part of an idealized, high-level, computational design" (ms.: 1). Such an understanding of choice theory does not seem to yield the fully determinate notion of an option Maier is after to begin with. That is because

[s]uch a model (...) could be a descriptive model, trying to capture, to a first approximation, the choices made by actual humans. Or it could be a normative model, specifying the choices people should ideally make. Or it could be a constitutive model, implicitly defining what it is to be an agent with such-and-such beliefs and desires. On each interpretation, we can't assume that the available acts are simply given as part of a well-defined decision problem. Somehow the agent's cognitive system must itself figure out the available options. (ibid.:1)

The psychological model of choice theory does not seem to be what Maier needs, then. Instead, options are supposed to be something objective. The agentive paths that are objectively open to the agent at a given time, or something along those lines.

I am not sure that this notion is as clear and well-understood as Maier seems to. Schwarz, at any rate, puts a lot of effort into spelling out what it is to have an option in the objective sense. And what he says will not please Maier, because, first, it does not treat the modality of options as modally fundamental, and, as we will see shortly, it does not sit well with Maier's ideas about reducing abilities to options. Schwarz writes that 
we could define objective options (for an agent at a time) as things the agent would do if she intended (at the time) to do them. I prefer a slightly weaker alternative that retains the existential form of our first stab: A is an objective option if some variation of the agent's intentions (at the time) would bring about A. (Schwarz, ms.)

In a nutshell, the view seems to be this:

$\mathrm{CA}_{\text {OPTIONS }}$ S $\mathrm{S}$ has an option if and only if there is some variation of the agent's intentions $\mathrm{V}$, such that, were $\mathrm{V}$ to occur, it would bring about $\phi$.

Of course, the careful reader will immediately balk at this: what about coma patients? What about phobics? Doesn't $\mathrm{CA}_{\text {OPTIONS }}$ yield wrong results in those cases? If the coma patient intended to raise her arm, she would. If the phobic intended to touch the spider, she would. Hence, $\mathrm{CA}_{\mathrm{OPTIONS}}$ seems to yield that the coma patient has the option of raising her arm the phobic has the option of touching the spider, respectively. But that seems wrong. Those agents don't seem to have those options.

Schwarz is well aware that demurring statements like this will be put forward by those engaged with abilities. But he does not think they matter to his project. Instead, he points out that his view of options will not be "adequate as a general analysis of the English auxiliary 'can”" (ibid.). But that is okay: decision theory is a completely different business, on his view. Coma patients, for instance, simply fall outside the scope of choice theory altogether:

decision-theoretic models describe the workings of an idealized cognitive system. They don't apply to rocks or corpses or people in a coma. Decision theory is not meant to predict or evaluate the behaviour of these systems, so we may well set aside the question how to delineate their options."(ibid.)

And phobics? They do actually have the option to do what they are phobic of on Schwarz's account. There is what he calls a "disconnection between decisiontheoretic options and everyday normative attitudes towards people's actions" (ibid.). Thus, when we are considering what agents can do, in terms of an everyday sense of "can", "we often hold fixed various facts that should not be held fixed when we catalogue the space of decision-theoretic options" (ibid.), according to Schwarz. For instance,

we exclude options that would go against deeply ingrained fears or hopes or convictions (...). Perhaps we can even hold fixed quite commonplace intentions: since you intend to change lanes, you ought to accelerate (...). There is also a temptation to hold fixed the past and the laws of nature, which is why some people intuit that determinism renders praise and blame pointless: you couldn't really have done anything else. In these respects, 
the range of options that figure in everyday normative evaluations is often more narrow than decision-theoretic options. (ibid.)

This is bad news for Maier. Apparently, it is far from clear that options, as they figure in choice theory, are the kinds of animals to which abilities can be successfully reduced. If a phobia does not deprive an agent of the option to touch a spider, because we don't hold the agent's deeply engrained fears fixed, then it is hard to see how it can possibly deprive her of the general ability to touch a spider, on Maier's view. Holding the phobia fixed, the agent will still have the option to touch spiders pretty much all the time. Thus, Maier's view yields that she also has the general ability. That is obviously mistaken. As far as I can see, the disconnection between choice theoretic options and abilities more generally, which Schwarz points to, is a genuine problem for Maier's account.

There is another problem with using the choice theoretic notion of an option as a fundament of an account of abilities more generally. The notion of an option, as it figures in choice theory, is such that it automatically renders having multiple options compatible with determinism. Witness Schwarz again:

the kinds of choices studied in decision theory (...) do not require a strong, libertarian kind of freedom. Decision theoretic algorithms are widely used in artificial intelligence, where it is taken for granted that the (artificial) agent's actions can be predicted from its internal architecture and the inputs it receives. If you build a robot, you don't need to specify that it should drop towards the Earth when it falls out of a helicopter. It will do that no matter what internal states and decision rules you build in. Not so when the robot reaches a junction. Here the outcome is under control of the robot's internal state: vary the state, and the robot will choose different paths. That is all the freedom we need. (ibid.)

What Schwarz points out in this passage is that options can be had, even if the actual future behavior of the system is determined. That is a problem for Maier because there seems to be a clear sense in which agents cannot do otherwise, if determinism is true. If all there is to abilities is options in varyingly large sets of circumstances, then no such sense can be spelled out. Holding determinism fixed, there will still be loads of options in the choice theoretic sense Maier uses in his account. And thus, there is no sense in which a predetermined path deprives agents of their abilities to act otherwise.

This is particularly problematic because the quest for a viable account of abilities has always been motivated, in part, by the more overarching quest for a more thorough understanding of the free will debate, with the compatibility problem at its center. There is obviously a sense in which agents cannot act otherwise in a deterministic world, and a sense in which they can. Which sense is relevant to the free will problem? This question is one that Maier will not be 
able to tackle with his account of abilities, which already presupposes compatibilism.

Finally, let me note a third problem with an understanding of general abilities in terms of options. It is hard to see how to integrate non-agentive abilities into the framework. I surely have the ability to smell, but I am not sure there are any situations in which I have smelling as an option in the sense in which options figure in choice theory. Options, I should think, are essentially options to perform actions. Non-agentive abilities are by definition abilities to exhibit behavior that does not qualify as actions, though. There seems to be a tension here, to say the least.

Of course, Maier will say that his view is intended as an account of agentive abilities only. And that response is perfectly respectable, of course. But, and here comes the qualification, the response is respectable only as long as we can think of a principled way of extending the framework also to cover non-agentive abilities.

In the case of the success view, the account of agentive abilities turned out to be a special instance of a broader scheme, which covers both agentive and nonagentive abilities. In the case of Maier's view, in contrast, it is not too easy to see what such an extension might possibly look like. If (i) the modality of options is irreducible and basic, (ii) Maier's account of agentive abilities is built upon those basic modal properties and (iii) options are the wrong kinds of animals to feature in an account of non-agentive abilities, then an account of non-agentive abilities will inevitably feature different modal properties as their fundament. Agentive and non-agentive abilities will then differ in the kind of modality that underlies them. That is a genuine problem, since the challenge is precisely, I take it, to come up with a view that accounts for the fact that the modality of agentive and non-agentive abilities seems to be of the same kind.

We can note, then, that Maier's account faces some severe difficulties, even though it obviously shares some important features with the success view. The problematic part, I have argued, is the attempt to reduce abilities in general to the choice theoretic notion of an option. Doing so plausibly yields wrong verdicts about phobics and other psychologically impaired agents, it fails to yield the obvious sense of ability, according to which an agent is unable to act otherwise, if determinism is true, and it yields problems when it comes to the integration of non-agentive abilities. I take it that these are three strong reasons to try and get along without the choice theoretical notion of an option. 


\subsection{Upshot}

In this chapter, we situated the success view within the contemporary literature on abilities and among some potential views that result from mapping some of the most promising recent contributions in the literature on dispositions onto the ability realm. As we saw, all of the recent accounts operate with the notion of proportions of worlds or situations. And most of them (Maier's option-based view excluded) spell out the same idea that also underlies the success view: that having an ability is a matter of a sufficient performance rate across a portion of the modal realm. In their broad outlines, then, the success view and the other views that were discussed in this chapter do not differ too much. As always, though, the devil is in the details. Looking more closely at each of the views, their more subtle relations to the success view become apparent.

Greco's (2007) view, I argued in section 6.1, is fully compatible with the success view, in that it states, roughly, that abilities are a matter of success across a sufficient proportion of the relevant possible situations, which is the core idea of the success view as well. What Greco does not say is how this is to be spelled out; here, the success view adds an important layer. According to the success view, success across a sufficient proportion of the relevant possible situations is for S-triggers of performances and the corresponding performances to match up across a sufficient proportion of the relevant possible situations. Greco, I said, can thus be seen as a 1.0 version of the success view (but also of other views, which can be seen as attempts to spell out what success across a sufficient proportion of the relevant possible situations amounts to).

In section 6.2, we looked in detail at what I called the sophisticated conditional analysis, versions of which have been proposed by Manley and Wasserman (2008) for dispositions, and by Sosa (2015) and Vihvelin (2011) for abilities. According to the sophisticated conditional analysis, having an ability is a matter of a sufficient proportion of counterfactuals of the form "If $\mathrm{C}$ were the case and $\mathrm{S}$ intended to $\phi$, S would $\phi$ " turns out true, where C picks out a highly specific set of circumstances.

The sophisticated conditional analysis is very similar to the success view in many ways. So similar, in fact, that once it is ameliorated in such a way that cases of impeded intentions can be accounted for, it really just is the success view in counterfactual guise. However, and this is really important if you have strong inclinations towards a counterfactual account of abilities, there is good reason to favor the non-counterfactual formulation of the success view. The counterfactuals figuring in the sophisticated conditional analysis may look like counterfactuals all right. Really, though, their antecedents are so specific that they pick out particular situations. Thus, the counterfactual appearance of 
the sophisticated conditional analysis therefore turns out to be a scam on a closer look and certainly not the most straightforward way of spelling out the thought that is supposed to be captured in the view.

While sections 6.1 and 6.2 dealt with views that can be seen as allies of the success view, I used sections 6.3 and 6.4 to discuss problems with two other views that seem rather appealing at first.

In section 6.3, I looked at a view which is obtained by analyzing abilities along the lines of Vetter's (2015) account of dispositions. The resulting view, which I called possibilism+, resembles the success view closely in that the only difference between the two views is that possibilism+ drops the restriction to intention situations that figures so prominently in the success view. According to possibilism+, having an ability to $\phi$ is a matter of $\phi$-ing in a sufficient proportion of the relevant possible situations, full-stop. No further restriction to intention situations is postulated.

The view looks appealing at first, because it is obviously somewhat simpler than the success view. As we saw, however, the view runs into trouble with cases in which agents exhibit an increased tendency to $\phi$ in virtue of an increased tendency to intend to $\phi$. The additional layer of complexity provided by the success view is therefore highly justified. Abilities really seem to be a matter of the modal tie between intentions and performances and not just of the sheer proportion of performance cases as such.

In section 6.4, finally, we looked at Maier's (2013) option-based account of abilities. On Maier's view, having an ability is a matter, not of $\phi$-ing, but of having the option to $\phi$ across a sufficient proportion of the relevant possible circumstances. Options are irreducible, on his view, but well-understood in virtue of their role in choice theory. I have laid out multiple problems with this view.

First, the notion of an option is not obviously as well-defined as Maier seems to think. Secondly, it is not clear that an account of abilities in terms of options yields the right verdicts in cases of impeded intentions. Thirdly, Maier's view seems incompatible with there being a good sense of having the ability to act otherwise, according to which that ability is incompatible with determinism. Finally, the view does not sit well with non-agentive abilities. I take these points to show that Maier's option-based view of abilities is inferior to the success view in some important ways. 